\title{
Index Assignment Optimization for Digital Communication Systems Using M-ary Modulation Schemes
}

\author{
Tran Ngoc Tuan, Nguyen Quoc Trung \\ School of Electronics and Telecommunications, Hanoi University of Science and Technology \\ Email: tuan.tranngoc@hust.edu.vn, trung.nguyenquoc@hust.edu.vn
}

Abstract - Channel-optimized Index Assignment (IA) of source codewords is a simple but effective approach of improving the error resilience of the communication systems. Although IA is a type of Joint Source Channel Coding (JSCC), it does not intervene with the source codec design. So, in addition to the fact that this method can be used in designing systems effectively, it can be also applied to the existing system. However, these prior IA methods have usually been based on assuming a Binary Symmetric Channel (BSC) and/or single-bit error in the codeword. These assumptions are only valid when the modulation is binary or the noise level in the channel is low. In this paper, we study the IA problem in a more general case in which the modulation is 2Dsignalling $M$-ary ( $M$-ary PSK, $M$-ary QAM), proposing the method to optimize IA for this case. Some simulations results based on this method are also given.

Keywords - Joint Source-Channel Coding, Index Assignment, Vector Quantization, Channel-Optimized Source coding ...

\section{INTRODUCTION}

In digital communication systems transmitting continuous amplitude sources, the source signal is quantized and encoded into the bitstream before being transmitted to the receiver. The encoding process usually includes source coding (data compression) and channel coding (error protection). Shannon's separation theorem of source and channel coding states that these two coding modules can be treated separately without any loss of performance assuming that both transmitter and receiver are permitted to have an unlimited complexity and delay [1]. Unfortunately, complexity and delay are two important restrictions for real-time applications and especially in two-way communications. As a result, it is advantageous to design the source and channel codes jointly, as witnessed by a large body of literature on joint source channel coding (JSCC). For a review of various JSCC techniques, see [6].

Two main schemes of JSCC is concatenated coding and combined source-channel coding. In concatenated coding scheme, the source and the channel coding blocks are separated but jointly optimized to provide a minimal end-to-end distortion or error probability. Another form of JSCC is combined source-channel coding, where the source coder is optimized according to the channel conditions.

There are two major approaches within this class of JSCC. In the first approach, the quantizer is modified taking into account the characteristic of the noisy channel and minimizing distortion caused by quantization and channel errors. This approach is referred to as Channel-Optimized Quantization (COQ) and includes both scalar and vector quantization [12]. However, it requires long training time and the exact knowledge of channel characteristics, which may not be always be avaiable. In addition, this scheme is only works effectively at the noise level used in the optimization. The second approach to combined source channel coding is optimization of Index Assignment (IA). This method rearranges the order of quantized source symbols in the optimized order, and works either with or without Forward Error Correction (FEC) coding.

In this paper, we adopt the IA approach since this is a simple but highly effective and feasible method. It is possible to reduce the sensitivity of the bit stream to errors without adding redundant bits and increasing the coder complexity. This approach can be employed as a method for improving the error resilience of pre- 
existing systems, while keeping other components intact.

An early design of IA is the Gray code [8] which labels two consecutive scalar source codewords by two binary numbers of Hamming distance one. In the general case, for a source code of fixed length $\mathrm{n}$ bits, there are $N=2^{n}$ codewords and $N$ ! possibilities to order $N$ codewords. To test $N$ ! assignments is a NPhard problem, which makes practically impossible finding an optimal solution for codebooks larger than 32 entries. For this reason, a number of different IA approximate solutions have been proposed [13-18].

Most works on this approach [13-21] are based on assuming a binary symmetric channel (BSC) without considering the modulation scheme. Meanwhile, with such assumptions, it is only valid for some special cases (binary modulation or low channel noise), but not the case $M$-ary modulation schemes when $M$ is large.

There are also some JSCC methods reported in the literature with regard to the modulation scheme. They either require the change in the constellation diagram $[9,10]$ or only consider the special case when a codeword is corresponding to a modulated symbol [11]. Once the constellation diagram is modified, it is necessary to re-design the modulation and demodulation unit with unusual constellation diagrams. Furthermore, it is possible that the modified modulation scheme is optimal for a certain codeword but not optimal for other codewords in the frame.

To address above limitations, we have proposed a novel IA method combined with the given modulation schemes for the case of $M$-ary modulation scheme. The proposed method has been proved and compared with the previous IA method by simulations.

The rest of the paper is organized into 5 sections. In Section II, the problem of optimal Index Assignment is fomulated. We then discuss the limitations of previous IA methods and propose a new IA method in Section III. The performance of the proposed method is evaluated by simulations in Section IV. And finally, conclusions are given in Section V.

\section{INDEX ASSIGNMENT PROBLEM STATEMENT}

A basic element of digital communication systems is the Quantizer Q, either scalar or vector. In this paper, we consider IA of Vector Quantization (VQ) because this is the general case of scalar quantization. Furthermore, VQ is an effective and widely-used method for low-bit-rate coding of high correlation signals such as speech, audio, image and video [2]. Specifically, most of the recent speech coder standards employ VQ technique.

When a set of discrete-time amplitude values is quantized jointly as a single vector, the process is known as Vector Quantization (VQ) [2], also known as block quantization. The block diagram of a Vector Quantizer is shown in Fig. 1. VQ encodes each vector from a sequence of source vectors with a channel symbol - a binary word chosen from a finite set. A typical VQ system contains a finite predetermined collection of codevectors (a codebook), and a vector distortion measure which, when given two vectors, yields a distance (or distortion) between them. At the encoder, the input vector is compared to each codevector in order to find the closest match and a binary index is transmitted to the decoder in order to inform about the selected codevector.

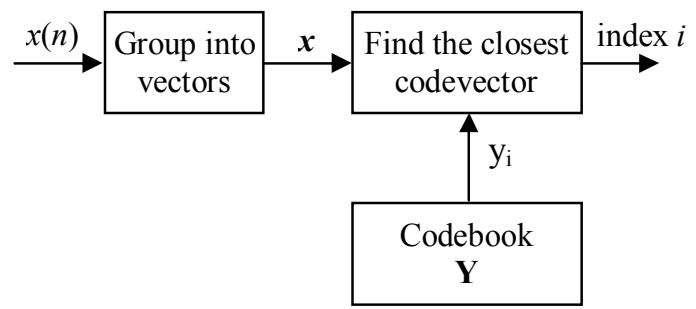

Fig. 1. Block diagram of a vector quantizer

The codebook design process is also known as trainning or populating the codebook. A well known algorithm for VQ codebook design is the Linde-BuzoGray (LBG) algorithm [4,7].

A vector quantizer $Q$ of dimension $K$ and size $N$ can be defined as a mapping of $K$-dimensional Euclidean space $R^{K}$ into a finite subset $Y$ containing $N$ vectors of $R^{K}$ given by:

$$
\begin{aligned}
Q: R^{K} & \rightarrow Y \\
x & \rightarrow y_{i}
\end{aligned}
$$




\section{Research, Development on Information and Communications Technology}

The codebook $Y=\left\{y_{i}, 1 \leq i \leq N\right\}$ is the set of $K$ dimensional codevectors, also known as reconstruction vectors or quantization vectors. The output of the vector quantizer is the index $i$ of the codevector $y_{i}$ which satisfies:

$$
i=\underset{k}{\operatorname{argmin}} d\left(x, y_{k}\right)
$$

where $d\left(x, y_{\mathrm{k}}\right)$ is the nonnegative distance between two vectors and a widely used distortion measure is the squared Euclidean distance, given by:

$$
\int d(x, y)=\|x-y\|^{2}
$$

The channel noise will include channel errors in communication. The effect of channel errors is to cause errors in the received indices which can result in significant distortion in decoded vectors.

Let $P(i)$ and $d(i, j)$ denote the probability of sending codevector $c_{i}$ and the distortion (or distance) between codevector $c_{i}$ and $c_{j}$. $P_{C}(j, i)$ represents the probability that the codeword (index) $j$ is received given the codeword $i$ is transmitted over the channel. The sum of possible distortions when transmitting vector $c_{\mathrm{i}}$ is:

$$
D_{i}(\pi)=P(i) \sum_{j=1}^{N} P_{C}(\pi(j), \pi(i)) d(i, j)
$$

where the IA function $\pi$ is a permutation of the integers $\{1,2, \ldots, N\} ; \pi(i)$ assigns an index to the $i$-th codevector.

The overall distortion is given by:

$$
D(\pi)=\sum_{i=1}^{N} P(i) \sum_{j=1}^{N} P_{C}(\pi(j), \pi(i)) d(i, j)
$$

Different index assignments do not change the distortion of the source code, but they do affect the overall distortion of a communication system $D(\pi)$ in case of channel error. Therefore, IA can be optimized with respect to channel statistics to mitigate the impact of channel error.

To avoid repeated evaluation of $P(i), P_{C}(i, j)$ and $d(i, j)$, all needed values can be pre-calculated and stored in matrices $\mathbf{P}_{\mathbf{C}}$ and $\mathbf{d}$ :

$$
\begin{aligned}
& \left\{\mathbf{P}_{\mathbf{C}}\right\}_{i, j}=P_{C}(i, j) \\
& \{\mathbf{d}\}_{i, j}=P(i) d(i, j)
\end{aligned}
$$

Then, Eq.(5) can be rewritten as:

$$
D(\pi)=\sum_{i=1}^{N} \sum_{j=1}^{N}\left\{\mathbf{P}_{\mathbf{C}}\right\}_{\pi(j), \pi(i)}\{\mathbf{d}\}_{i, j}
$$

The IA problem is to find the best codebook rearrangement $\pi$ which minimize $D(\pi)$ for given input parameters $\mathbf{P}_{\mathbf{C}}$ and $\mathbf{d}$. $D(\pi)$ is also called the objective function in combinatorial optimization problems.

The matrix d can be calculated from the codebook and the training set. Meanwhile, the codeword transition probability matrix $\mathbf{P}_{\mathbf{C}}$ depends on the channel characteristics and can be evaluated by theoretical model. In case the channel is BSC, the probability function $P_{C}(i, j)$ is given by:

$$
P_{\mathbf{C}}(i, j)=\varepsilon^{h(i, j)}(1-\varepsilon)^{n-h(i, j)}
$$

where $h(i, j)$ denote the Hamming distance (number of bit differences) between $i$ and $j$. In this case, the $\mathbf{P}_{\mathbf{C}}$ matrix is always symmetric.

\section{THE OPTIMAL IA METHOD FOR M-ARY MODULATION SCHEMES}

Most the optimal IA methods in previous works do not take into account the modulation scheme and the $\mathbf{P}_{\mathbf{C}}$ matrix is computed by ( 8 ) on the assumption of BSC channel. This assumption is only valid when the modulation scheme is binary or QPSK with Gray coding, but in the case of $M$-ary modulation, the above calculation of $\mathbf{P}_{\mathbf{C}}$ is only approximate in nature.

In this section, we propose a novel optimal IA method for the case of $M$-ary modulation. The proposed method adopts existing IA algorithms such as binary switching algorithm (BSA) [13], simulated annealing algorithm $[14,18]$, Tabu search [15] to find the suboptimal IA. However, with our method, the $\mathbf{P}_{\mathbf{C}}$ matrix is calaculated exactly instead of approximately by Eq.(8), so the system can achieve better performance.

The exactly calculation of $P_{C}(a, b)$, the probability of transmitting codeword $b$ but receiving codeword $a$, is presented in the rest of this section. We also propose the algorithm to exactly compute the probability $P_{\mathrm{C}}(a, b)$ in Section 3.2. Hence, we can obtain the entire $\mathbf{P}_{\mathbf{C}}$ matrix for the IA optimization problem. 


\subsection{System model and parameters.}

We consider the fixed-length codeword $b$ transmitted via the communication system, and the codeword $a$ is received after decoding at the receiver. Before being transmitted, the codeword $b$ is assembled into a frame together with other codewords and data bits. Assuming that the frames are transmitted in a row by the $M$-ary digital modulator and the codeword being considered occupies $n$ consecutive bits of the frame, the bits of the frame are allocated into symbols and each symbol is assigned a waveform of a set of $M$ waveforms for transmission over channel. Let $l, n, m$ denote the length (number of bits) of the frame, the codeword $b$ (and $a$ ) and the modulation symbol respectively.

Fig.2 describes all different possibilities for allocating the $n$-bit codeword $b$ into symbols of length $m$ bits. For the $k$-th allocation possibility, the codeword $b$ is allocated into $n_{s k}$ symbols $s_{k 1}^{(b)}, s_{k 2}^{(b)}, \ldots, s_{k n_{k k}}^{(b)}$, and similarly, the codeword $a$ is also represented by $n_{\mathrm{sk}}$ symbols $s_{k 1}^{(a)}, s_{k 2}^{(a)}, \ldots, s_{k n_{s k}}^{(a)}$.

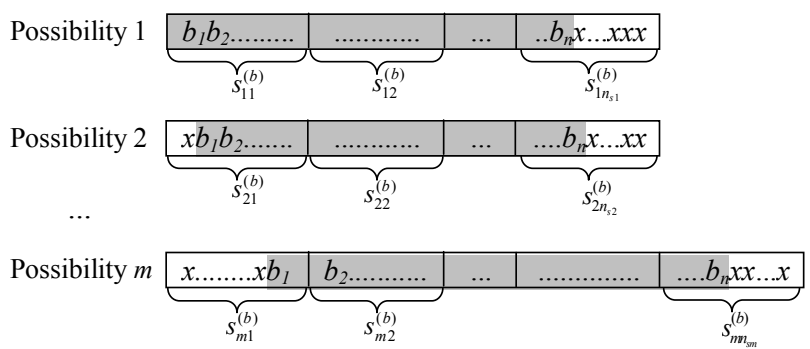

Fig. 2. Possibilities for allocating the n-bit codeword $b_{1} b_{2} \ldots b_{n}$ into m-bit symbols

Let $p_{n}$ be the number of possibilities for allocating the codeword $b$ into symbols. From the Fig.2, it can be seen that the maximum value of $p_{n}$ are $m$. In the case that the codeword $b$ is not totally allocated into a whole number of symbols, the first symbol $s_{k 1}^{(b)}$ and the last symbol $s_{k n_{*}}^{(b)}$ can include $x$ bits which are bits in the frame but not belong to the codeword $b$.

In order to calculate $P_{C}(a, b)$, we have to determine the codeword transition probability of all the above allocation possibilities. The probability corresponding to the $k$-th allocation possibility $P_{C_{k}}(a, b)$ is the product of $n_{s k}$ probabilities:

$$
P_{C_{k}}(a, b)={\stackrel{\bigcirc}{\tilde{O}_{k}}}_{i=1}^{n_{k i}} P_{k i}^{(a b)}
$$

where $P_{k i}^{(a b)}$ is the probability of transmitting symbol $s_{k i}^{(b)}$ but receiving symbol $s_{k i}^{(a)}$ :

$$
P_{k i}^{(a b)} \triangleq P\left(s_{k i}^{(a)} \mid s_{k i}^{(b)}\right)
$$

The symbol transition probability for transmittion over AWGN channels and fading channels with arbitrary 2-D signaling can be exactly computed in [3]. To facilitate the calculation, all the symbol transition probabilities of $M$ symbols of the modulation scheme being considered can be precalculated and stored in the matrix $\mathbf{P}_{\mathbf{S}}$ :

$$
\left\{\mathbf{P}_{\mathrm{s}}\right\}_{s_{r}, s_{t}}=P\left(s_{r} \mid s_{t}\right) \quad 0 \leq s_{t}, s_{r} \leq M-1
$$

If symbols $s_{k i}^{(a)}$ and $s_{k i}^{(b)}$ do not contain $x$-bits, $P_{k i}^{(a b)}$ can be taken directly from the matrix $\mathbf{P}_{\mathbf{S}}$. In case $s_{k i}^{(a)}$ and $s_{k i}^{(b)}$ contain $x$-bits, then in order to evaluate $P_{k i}^{(a b)}$, all possibilities of these $x$-bits need to be considered. Assumming the first $r_{1}$ bits and the last $r_{2}$ bits of symbols $s_{k i}^{(a)}$ and $s_{k i}^{(b)}$ are $x$-bits, the binary form of these symbols are:

$$
\begin{aligned}
& s_{k i}^{(a)}=\underbrace{x x \ldots x}_{c_{x_{a}\left(r_{1}\right. \text { bit }}} \underbrace{a_{1} a_{2} \ldots a_{n}}_{c_{a}} \underbrace{x x \ldots x x}_{c_{x_{a} 2}\left(r_{2} \text { bit }\right)} \\
& s_{k i}^{(b)}=\underbrace{x x \ldots x}_{c_{x_{b 1}}\left(r_{1} \text { bit }\right)} \underbrace{b_{1} b_{2} \ldots b_{n}}_{c_{b}} \underbrace{x x \ldots x x}_{c_{x_{b 2}}\left(r_{r_{2}} \text { bit }\right)}
\end{aligned}
$$

Note that $c_{x_{a 1}}, c_{x_{a 2}}, c_{x_{b 1}}, c_{x_{b 2}}, c_{a}, c_{b}$ are the decimal representation of the corresponding bit groups. The above symbols can be represented in decimal form as:

$$
\begin{gathered}
s_{k 1}^{(a)}=2^{r_{2}+n} c_{x_{a 1}}+2^{r_{2}} c_{a}+c_{x_{a 2}} \\
s_{k 1}^{(b)}=2^{r_{2}+n} c_{x_{b 1}}+2^{r_{2}} c_{b}+c_{x_{b 2}}
\end{gathered}
$$

The probability $P_{k i}^{(a b)}$ can be calculated as follows:

$$
\begin{aligned}
& P_{k i}^{(a b)}=\frac{1}{2^{r_{1}+r_{2}}}, \\
& 2^{n}-12^{n_{2}}-12^{n}-12^{n_{2}}-1
\end{aligned}
$$

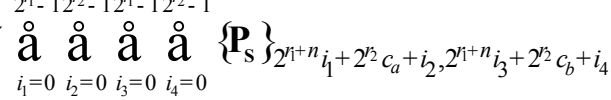




\section{Research, Development on Information and Communications Technology}

From the system model, the above parameters and analyses, we propose the algorithm of evaluating $P_{C}(a, b)$ in Section 3.2. With this algorithm, we can calculate the entire matrix $\mathbf{P}_{\mathbf{C}}$ for the IA problem.

\subsection{Algorithm of evaluating $P_{C}(a, b)$ for optimizing of Index Assignment.}

The proposed algorithm of evaluating $P_{C}(a, b)$ is divided into 4 steps described as follows:

Step 1: Depending on the position of the codeword in the frame and the parameters $n, m, l$, determining all possibilities of allocating the codeword into modulation symbols (similar to the description in Fig.2). In case the frame length $l$ and the position of the codeword within the frame are fixed, the number of possibilities is:

$$
p_{n}=\frac{m}{\mathrm{GCD}(m, l)}
$$

where $\operatorname{GCD}(m, l)$ is the Greatest Common Divisor of $m$ and $l$. When the frame length $l$ or the position of the codeword in frame is not fixed, we need to investigate all probable cases for each of these factors. If these factors cannot be anticipated, all possible allocation possibilities need to be examined in this step and then the number of possibilities is maximum $p_{n}=m$.

Step 2: For each possibility in the Step 1, evaluate all symbol transition probabilities $P_{k i}^{(a b)}$ with $0 \leq i \leq n_{s k}$ and $1 \leq k \leq c_{\max }$.

If all symbols $s_{k i}^{(a)}$ và $s_{k i}^{(b)}$ do not include $x$-bits, $P_{k i}^{(a b)}$ can be obtained directly from matrix $\mathbf{P}_{\mathbf{s}}$.

$$
P_{k i}^{(a b)}=\left\{\mathbf{P}_{\mathbf{S}}\right\}_{s_{k i}^{(a)}, s_{k i}^{(b)}}
$$

In case symbols $s_{k i}^{(a)}$ and $s_{k i}^{(b)}$ include $x$-bits, the $x$-bits group can be at the beginning, at the end or at both the beginning and the end of the symbol. We consider three cases as follows:

+ Case 1: The $x$-bits are at the beginning of the symbol. This case can occur at the first symbol (when $i=1$ ), the binary forms of $s_{k 1}^{(a)}$ and $s_{k 1}^{(b)}$ are :

$$
\begin{aligned}
& s_{k 1}^{(a)}=\underbrace{x_{\ldots}}_{r \text { bit }} \underbrace{a_{1} a_{2} \ldots a_{m-r}}_{c_{a}} \\
& s_{k 1}^{(b)}=\underbrace{x \ldots x}_{r \text { bit }} \underbrace{b_{1} b_{2} \ldots b_{m-r}}_{c_{b}}
\end{aligned}
$$

This is a specific case of the case considered in section 3.1, substitute $r_{1}=r$ and $r_{2}=0$ into Eq.(14) we get:

$$
P_{k 1}^{(a b)}=\frac{1}{2^{r}} \underset{i_{1}=0}{\stackrel{2^{r}-12^{r}-1}{a}}\left\{\mathbf{P}_{i_{2}=0}\right\}_{2^{r} i_{1}+c_{a}, 2^{r} i_{2}+c_{b}}
$$

+ Case 2: The $x$-bits are at the end of the symbol. This case can occur at the last symbol $\left(i=n_{s k}\right), s_{k k_{s k}}^{(a)}$ and $s_{k n_{s k}}^{(b)}$ can be expressed in binary forms as follows:

$$
\begin{aligned}
& s_{k n_{s k}}^{(a)}=\underbrace{a_{1} a_{2} \ldots a_{m-r}}_{c_{a}} \frac{x_{r . \ldots x}}{r \text { bit }} \\
& s_{k n_{s k}}^{(b)}=\underbrace{b_{1} b_{2} \ldots b_{m-r}}_{c_{b}} \underbrace{x_{\ldots} \ldots x}_{r \text { bit }}
\end{aligned}
$$

Replace $r_{1}=0$ and $r_{2}=r$ into Eq.(14), we have:

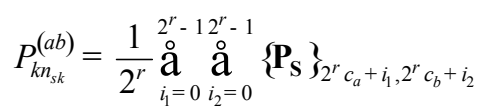

+ Case 3: The $x$-bits are at both the beginning and the end of the symbol. This case may occur when $n<m$, and codeword is only allocated in the only one symbol. The binary form of symbols are as follows:

$$
\begin{aligned}
& s_{k 1}^{(a)}=\underbrace{x x \ldots x}_{c_{x_{11}}\left(r_{1} \text { bit }\right)} \underbrace{a_{1} a_{2} \ldots a_{n}}_{a} \underbrace{a_{x} \ldots x}_{c_{x_{a 2}\left(r_{2} \text { bit }\right)}^{x x \ldots x x}} \\
& s_{k 1}^{(b)}=\underbrace{x x \ldots x}_{c_{x_{b_{1}}}\left(r_{1}\right. \text { bit) }} \underbrace{b_{1} b_{2} \ldots b_{n}}_{b} \underbrace{x x \ldots x x}_{c_{x_{b_{2}}}\left(r_{2} \text { bit }\right)}
\end{aligned}
$$

in which $c_{x_{a 1}}, c_{x_{a 2}}, c_{x_{b 1}}, c_{x_{b 2}}$ are the decimal representation of the corresponding bit groups. Substitute $c_{a}=a$ and $c_{b}=b$ into Eq.(14) we get:

$$
\begin{aligned}
& P_{k 1}^{(a b)}=\frac{1}{2^{r_{1}+r_{2}}} \cdot \\
& 2^{n}-12^{2}-12^{n}-12^{n}-1
\end{aligned}
$$

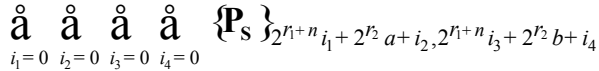

Step 3: Evaluate the codeword transition probability corresponding to each allocation possibility of Step 1. 
For the $k$-th possibility, $P_{C_{k}}(a, b)$ can be computed by substituting probabilities $P_{k i}^{(a b)}$ calculated in Step 2 into Eq.(9).

Step 4: Finally, the probability $P_{C}(a, b)$ is calculated by summarizing the probabilities of all possibilities. Normally, in case the frames are transmitted consecutively, all the allocation possibilities are equiprobable with probability $1 / p_{n}$.

$$
P_{C_{C}}(a, b)=\frac{1}{p_{n}} \stackrel{a}{k=1}_{p_{n}}^{p_{n}} P_{C_{k}}(a, b)
$$

\section{EXPERIMENTS AND DISCUSSION}

In this section, computational experiments are carried out in Matlab to examine the performance of the proposed method and to compare it with other methods.

\subsection{Experimental setup.}

We consider a communication system where the source encoder/decoder is a vector quantizer of size $N$ $(N=32$ and 128) and the modulation is square 16QAM $(M=16, m=4)$ and square 64-QAM $(M=64$, $m=6)$ with Gray encoding. The message emitted from the source is first partitioned into vectors of dimension $K=4$; the vector quantizer is then used to compress the input vectors; after that the indices corresponding to the input vectors are modulated to transmitting over the AWGN channel.

The VQ technique is generally applied to effectively encode the signals with high correlation. In order to model such correlated source generally, the the input signal tested is Gauss-Markov process with high value of the correlation coefficient $\rho$. The firstorder Gauss-Markov processes are of the form:

$$
x(n)=\rho x(n-1)+w(n)
$$

where $w(n)$ is a zero-mean, unit variance, Gaussian white noise process. The magnitude of $\rho$ is less than one and $\rho$ is set to 0.9 in our simulations.

Applying LBG algorithm [4] with training ratios (number of training vectors divided by $N$ ) of 1000 and squared Euclidean distance measure, codebooks of $N=32$ and 128 codevectors are generated. For each codebook, the SA algorithm [14,18], in which control parameters are the same as in [18], is applied to find the suboptimal IA corresponding to the two cases: the input parameter $\mathbf{P}_{\mathbf{C}}$ is determined by the original method (approximate evaluation using Eq.(8)) and by the proposed method (accuary evaluation). The $\mathrm{E}_{\mathrm{s}} / \mathrm{N}_{0}$ level used for IA optimization in our experiments is $5 \mathrm{~dB}$. The below sections 4.1.1 and 4.1.2 describe in more details these two methods.

\subsubsection{Optimization of IA with the proposed method.}

To implement the proposed algorithm in Section 3.2 , the symbol transition probability matrix $\mathbf{P}_{\mathbf{S}}$ of the modulation techniques square 16-QAM and 64-QAM under the conditions of AWGN channel and given noise levels is determined.

Subsequently, applying the proposed algorithm to determine the matrix $\mathbf{P}_{\mathbf{C}}$ for the four cases with input parameters listed in Table 1. Since the system is considered in the case of consecutive transmission of the $n$-bit codewords, each frame contains only one codeword (i.e. $l=n$ ).

Table 1. Input parameters of the four test cases

\begin{tabular}{|l|l|l|}
\hline & $M=16$ & $M=64$ \\
\hline \multirow{2}{*}{$N=32$} & $\begin{array}{l}n=l=5 \\
m=4\end{array}$ & $\begin{array}{l}n=l=5 \\
m=6\end{array}$ \\
\hline \multirow{2}{*}{$N=128$} & $\begin{array}{l}n=l=7 \\
m=4\end{array}$ & $\begin{array}{l}n=l=7 \\
m=6\end{array}$ \\
\hline
\end{tabular}

In all above four cases, $\operatorname{LCD}(m, l)=1$ proceeding as in (14), so the number of possibilities for allocating the codeword into symbols is maximum $p_{n}=m$. For brevity, we investigate only the case of $N=128$ and $M=16$ here, the other cases are similar. The evaluation of $P_{C}(a, b)$ according to the proposed algorithm in section 3.2 is carried out as follows.

Firstly, determine the possibilities for allocating the 7-bit codeword into 4-bit symbols. Fig. 3 describes 4 allocation possibilities, in which the number of symbols are: $n_{s 1}=n_{s 2}=2$ and $n_{s 3}=n_{s 4}=3$. 


\section{Research, Development on Information and Communications Technology}

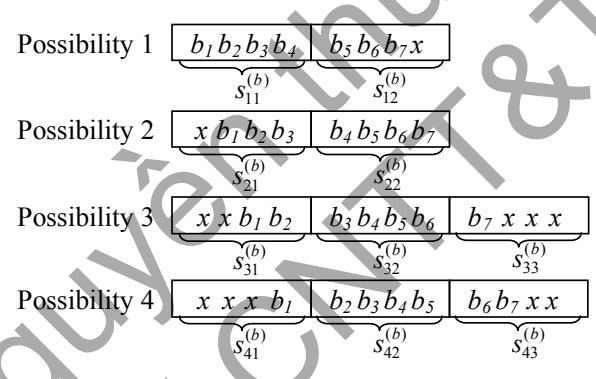

Fig. 3. Possibilities for allocating the 7-bit codeword into 4-bit symbols $(M=16, N=128)$

Then, the probabilities $P_{k i}^{(a b)}$ are calculated as follows:

- $P_{11}^{(a b)}, P_{22}^{(a b)}, P_{32}^{(a b)}, P_{42}^{(a b)}$ can be taken directly from

the matrix $\mathbf{P}_{\mathbf{S}}$ proceeding as in Eq.(16).

- $P_{21}^{(a b)}, P_{31}^{(a b)}, P_{41}^{(a b)}:$ case $3(i=1)$, using Eq. (18).

- $P_{12}^{(a b)}, P_{33}^{(a b)}, P_{43}^{(a b)}$ : case $2\left(i=n_{s k}\right)$, using Eq.(20).

Next, at the Step 3, substitute the values $P_{k i}^{(a b)}$ into Eq.(9) to compute $P_{C_{k}}(a, b)$.

Lastly, use Eq.(23) to calculate $P_{C}(a, b)$.

After establishing the above function, the matrix $\mathbf{P}_{\mathbf{C}}$ can be determined by using this function to calculate all its elements.

\subsubsection{Optimization of $I A$ with the original method.}

The bit error probability of squared 16-QAM and 64-QAM modulation with Gray coded constellation mapping in additive white Gaussian noise conditions can be evaluated as follows [5]:

$$
\begin{aligned}
\varepsilon_{b, 16 \mathrm{QAM}}= & \frac{3}{8} \operatorname{erfc}\left(\sqrt{\frac{\gamma_{s}}{10}}\right)+\frac{1}{4} \operatorname{erfc}\left(3 \sqrt{\frac{\gamma_{s}}{10}}\right)-\frac{1}{8} \operatorname{erfc}\left(5 \sqrt{\frac{\gamma_{s}}{10}}\right) \\
\varepsilon_{b, 64 \mathrm{QAM}}= & \frac{7}{24} \operatorname{erfc}\left(\sqrt{\frac{\gamma_{s}}{42}}\right)+\frac{1}{4} \operatorname{erfc}\left(3 \sqrt{\frac{\gamma_{s}}{42}}\right)-\frac{1}{24} \operatorname{erfc}\left(5 \sqrt{\frac{\gamma_{s}}{42}}\right)+ \\
& \frac{1}{24} \operatorname{erfc}\left(9 \sqrt{\frac{\gamma_{s}}{42}}\right)-\frac{1}{24} \operatorname{erfc}\left(13 \sqrt{\frac{\gamma_{s}}{42}}\right)
\end{aligned}
$$

where $\gamma_{s}=\mathrm{E}_{\mathrm{s}} / \mathrm{N}_{0}$ is the received channel signalto-noise ratio per symbol and $\operatorname{erfc}($.$) is the$ complementary error function.

Then, the $\mathbf{P}_{\mathbf{C}}$ matrix is evaluated by Eq.(8).

\subsection{Results and discussion}

The first experiment is performed to examine the accuracy of the proposed algorithm for calculating the codeword transition probability $P_{\mathrm{C}}(a, b)$.

Consider the case as described in Section 4.1.1, the values $P_{C}(a, b)$ are calculated by three methods: the proposed method (accuracy evaluation), approximate method and simulation. Fig.4 represents the values $P_{C}(a, b)$ evaluated by these three methods with two typical cases in which $a=b$ (error-free transmission) and $a \neq b$ with one bit diffirence.

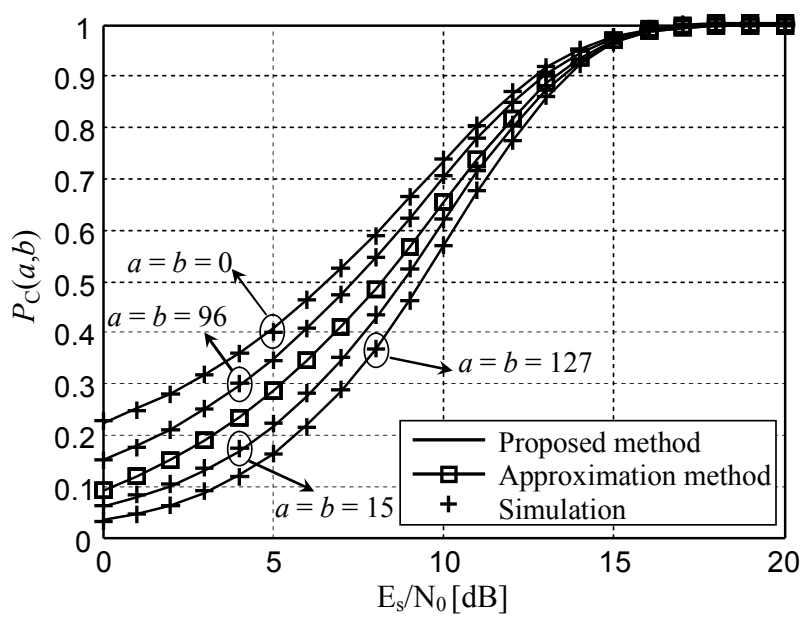

(a) $a=b$

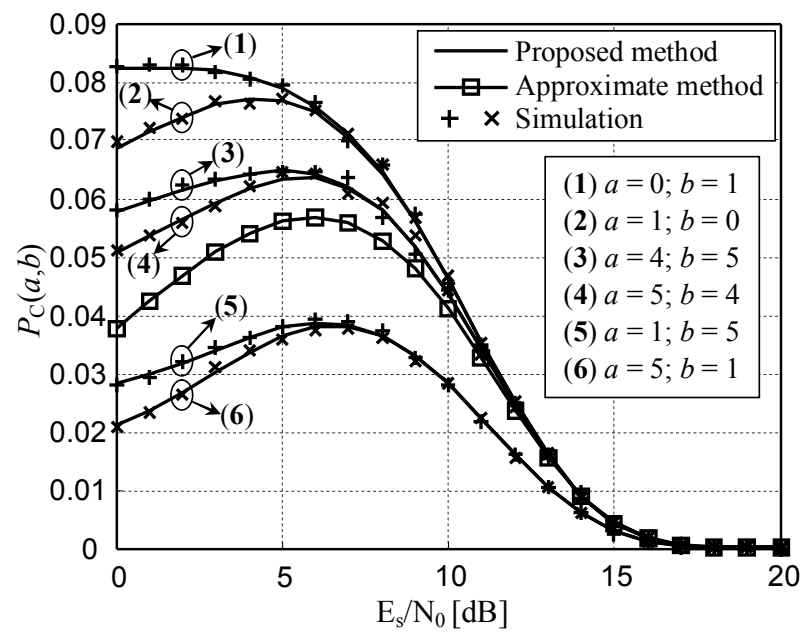

(b) $a \neq b$ (one bit diffirence)

Fig. 4. Codeword transition probability $P_{C}(a, b)$ evaluated by different methods (in the case of $N=128, M=16$ ) 
As seen from the Fig.4, the values $P_{\mathrm{C}}(a, b)$ evaluated by the proposed method agree well with the simulation results, this confirms the accuracy of the proposed algorithm. When evaluating $P_{C}(a, b)$ by the approximation method, with pairs of parameters $(a, b)$ which have the same Hamming distance, it will return the same result. Thus, there is only one approximation curve for all instances of $a$ and $b$ in Fig.4a and Fig.4b. It can also be seen that, at low to moderate $\mathrm{E}_{\mathrm{s}} / \mathrm{N}_{0}$, the exact values $P_{C}(a, b)$ are quite significant differences from the approximate values and are not equal to $P_{C}(a, b)$, i.e. the exact matrix $\mathbf{P}_{\mathbf{C}}$ is not symmetric as calculated by the approximation method. As a result, the IÂ method with accuary evaluation of $\mathbf{P}_{\mathbf{C}}$ is more effective than the original IA method with approximate evaluation of $\mathbf{P}_{\mathbf{C}}$, especially when the $\mathrm{E}_{\mathrm{s}} / \mathrm{N}_{0}$ is low to moderate.

The second experiment compared the performance of the entire system when using the different IA schemes with the same input signal source of 4,000,000 samples which are divided into 1,000,000 4-dimensional vectors. For each codebook tested, the signal-to-noise ratio (SNR) of the overall system defined as $\mathrm{SNR}=10 \log _{10}\left(\sigma_{x} / \sigma_{n}\right)[\mathrm{dB}]$, where $\sigma_{x}$ and $\sigma_{n}$ are the signal and noise variances respectively, is calculated with $\mathrm{E}_{s} / \mathrm{N}_{0}$ ranging from $0 \div 20 \mathrm{~dB}$.

The proposed method is compared with two other schemes, the original method and the random IA. With the proposed method and the original method, system is executed 10 times with 10 different IA schemes which are the results of 10 times running the SA algorithm. Then, the average SNR of 10 times execution is calculated for each method. Fig. 5 shows the average SNR of the overall system for each method against the $\mathrm{E}_{\mathrm{s}} / \mathrm{N}_{0}$.

From the Fig. 5, we observe that the system using the IA optimized by the proposed method outperforms other systems in increasing the SNR. However, compared with the approximation method, the gain of the proposed method becomes smaller at higher $\mathrm{E}_{\mathrm{s}} / \mathrm{N}_{0}$. The reason is that the difference between the matrix $\mathbf{P}_{\mathbf{C}}$ evaluated by exact method and by approximate method is neglibible at high $\mathrm{E}_{\mathrm{s}} / \mathrm{N}_{0}$.
When the $E_{s} / N_{0}$ is high enough, the channel can be assumed to be noiseless and all methods have similar performance at an ideal SNR. This ideal SNR level is also known as the Signal-to-Quantization Noise Ratio $\left(\mathrm{SN}_{\mathrm{q}} \mathrm{R}\right)$ and the $\mathrm{SN}_{\mathrm{q}} \mathrm{R}$ corresponding to the case $N=32$ and $N=128$ in this experiment are $11.6 \mathrm{~dB}$ and $14.4 \mathrm{~dB}$ respectively.

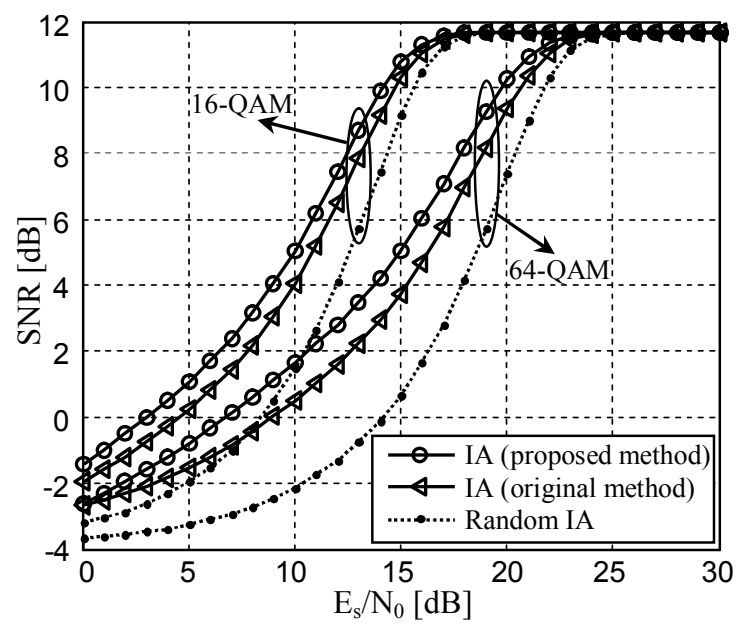

(a) $N=32$

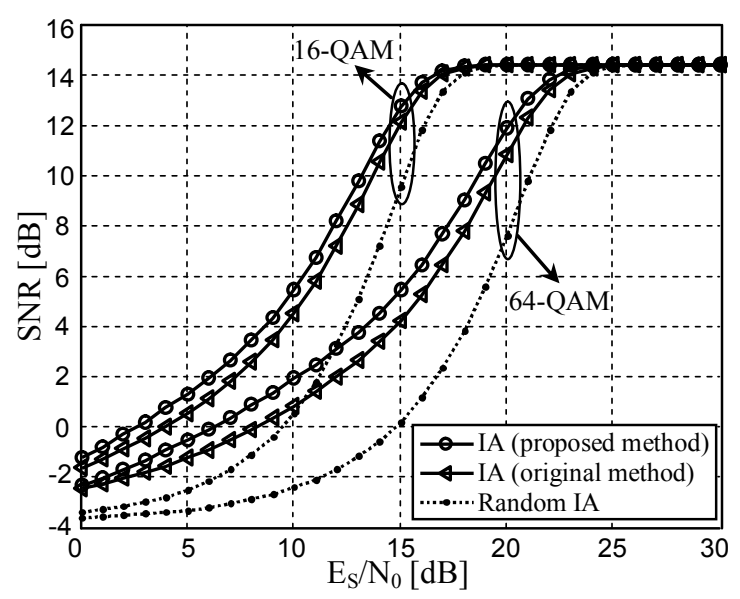

(b) $N=128$

Fig.5. Performance comparison of system using various IA schemes

At moderate $\mathrm{E}_{\mathrm{s}} / \mathrm{N}_{0}$, the gains of the proposed method with respect to the original method is about $0.8-1 \mathrm{~dB}$ for the case of 16-QAM modulation. In the case of 64-QAM modulation, the gain is higher and is about $1-1.3 \mathrm{~dB}$. This is because the differences between the codeword transition probabilities 
Research, Development on Information and Communications Technology

evaluated by the accurate method and the approximate method in the case of 64-QAM modulation is more than in the case of 16-QAM modulation, and the probability of error of 64-QAM is higher than 16QAM.

Through the above experiments, it proves the correctness and effectiveness of the proposed method.

\section{CONCLUSION}

In this paper, a new IA method is developed to improve the error resilience of the communication system transmitting continuous amplitude sources for the case that the modulation system is 2D-signalling $M$-ary. We study the general case when the codeword is transmitted in frame and the codeword is not necessary to be fully allocated into a whole number of modulation symbols as some prior works. An algorithm of exactly evaluating the codeword transition probability $P_{C}(a, b)$, the input parameters of the IA problem, is proposed for the new IA method. Then, the existing IA algorithms can be applied to optimize the system in this general case. In addition to applying to the IA problem, the proposed algorithm can also be applied to other related works about IA method and other JSCC approaches such as COVQ.

Theoretical analysis and simulations have shown that there is a significant difference between the exact codeword transition probability compared with the approximate value when the channel SNR level is low, it leads to the optimization of the system by the proposed method can achieve better performance than the system optimized by the previous approximation method.

Studies are in process to optimize more complex coding structures, such as in MultiStage VQ, Switched Split VQ, Switched MultiStage VQ. In addition, combining source channel coding and modulation scheme in more general cases and channel models would be one of future works.

\section{REFERENCES}

[1] C.E. Shannon, "A Mathematical Theory of Communication", Bell System Techical Journal, vol. 27, pp. 379-423, 623-656, 1948.
[2] A. Gersbo and R. Gray, "Vector quantization and signal compression", Boston, Ma. Kluwer Academic Publishers, 1992.

[3] L. Xiao and X. Dong, "The exact transition probability and bit error probability of twodimensional signaling", IEEE Trans. Wireless Commun., vol.4, pp. 2600-2609, Sep. 2005.

[4] Y. Linde, A. Buzo, and R. M. Gray, "An algorithm for vector quantization design", IEEE Trans. on Commun., Vol. COM-28, pp. 84-95, 1980.

[5] D. Yoon, K. Cho, J. LEE, "Bit error probability of Mary quadrature amplitude modulation", Vehicular Technology Conference, 2000. IEEE-VTS Fall VTC 2000. 52nd. IEEE, pp. 2422-2427, 2000.

[6] S. B. Z. Azami, P. Duhamel, and O. Rioul, "Joint source-channel coding: Panorama of methods," Proceedings of CNESWorkshop on Data Compression, pp 1232-1254, 1996.

[7] Tzu-Chuen Lu, and Ching-Yun Chang. "A survey of VQ codebook generation", Journal of Inf. Hiding and Multimedia Signal Processing, Ubiquitous International, Vol. 1, No. 3, pp. 190-203, 2010.

[8] F. Gray, "Pulse code communications", United States Patent Number 2632058, 1953.

[9] K. Ramchandran et al., "Multiresolution broadcast for digital HDTV using joint source/channel coding", IEEE Journal on Selected Areas in Commun., vol. 11.1, pp. 6-23, Jan. 1993.

[10] J.-K. Han and H.-M. Kim, "Joint optimization of VQ codebooks and QAM signal constellations for AWGN channels", IEEE Trans. on Commun., Vol. 49, Iss. 5, pp 816-825, 2001.

[11] H. Elmeddeb, et al, "Combined Adaptive Modulation and Channel Optimized Vector Quantization", IEEEEURASIP-ISCCSP, 2006.

[12] N. Farvardin and V. Vaishampayan, "On the performance and complexity of channel-optimized vector quantizers," IEEE Trans. Inf. Theory, vol. 37, no. 1, pp. 155-160, Jan. 1991.

[13] K. Zeger and A. Gersho, "Pseudo-Gray Coding”, IEEE Trans. on Commun., Vol. 38, No. 12, 2147-2158, 1990.

[14] N. Farvadin, "A Study of Vector Quantization for Noisy Channels", IEEE Trans. on Inf. Theory, Vol. 36, No. 4, 799-809, 1990.

[15] J.S. Pan, C.S. Shieh and S.C. Chu, "Comparison study on VQ codevector index assignment", Proc. 5th Int. Conf. Spoken Language Processing (ICSLP'98), Sydney, Australia, 1998, paper 0031.

[16] A. Miri, E. Hons and A.K. Khandani, "Optimising the combined source and channel coding of a discrete communication system". IEE Proc. Commun., vol. 152 , iss. 3, pp. 282-287, 2005. 
[17] W. Yue, “An Index Assignment Algorithm over Noisy Channel", Software Engineering and Knowledge Engineering: Theory and Practice, Springer Berlin Heidelberg, 2012, pp. 663-671

[18] T.N. Tuan, N.Q. Trung, "Improving the Simulated Annealing algorithm for the Index Assignment method to enhance the robustness of communication systems", Special Issues on Research, Dev. and Appl. on Inf. \& Commun. Tech., Vietnamese Journal on Inf. Tech. \& Commun., vol E-3, No. 7(11), pp. 13-20, 2014.

[19] W. Xiaohan and X. Wu, "Index Assignment Optimization for Joint Source-Channel MAP Decoding", IEEE Trans. on Commun., Vol 58.3, pp. 901-910, 2010.

[20] H. Oztoprak, S. Villette and A. Kondoz, "Index assignment-based channel coding", IET Commun., vol. 6, iss. 2, pp. 172-178, 2012.

[21] Xiaolin $\mathrm{Wu}$, et al, "On computation of performance bounds of optimal index assignment", IEEE Trans. on Commun., vol. 59, pp. 3229-3233, 2011.

\section{AUTHORS' BIOGRAPHIES}

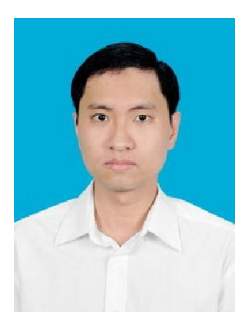

Tran Ngoc Tuan is working at School of Electronics and Telecommunications, Hanoi University of Science and Technology, Vietnam. He received the Engineer and M.Sc. degrees in electrical engineering from Hanoi University of Science and Technology in 2005 and 2008. His research interests include digital signal processing, speech coding and joint source channel coding.

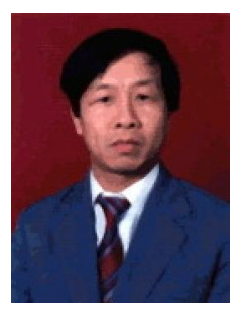

Nguyen Quoc Trung is working at School of Electronics and Telecommunications, Hanoi University of Science and Technology, Vietnam. $\mathrm{He}$ received Ph.D degree from Hungarian Academy of Science in 1982. He was promoted to Accociate Professor in 2004. His research interests include digital signal processing, subband coding and digital communication. 